nephron

Practice
Nephron 2018;138:22-28

DOI: $10.1159 / 000481182$
Received: May 23, 2017

Accepted after revision: July 20, 2017 Published online: October 20, 2017

\title{
Discrepancies between Perceived and Measured Cognition in Kidney Transplant Recipients: Implications for Clinical Management
}

\author{
Aditi Gupta $^{a}$ b Tashra S. Thomas ${ }^{a}$ Jeffrey A. Klein ${ }^{a}$ Robert N. Montgomery ${ }^{b, c}$ \\ Jonathan D. Mahnken $^{b, c}$ David K. Johnson ${ }^{b, e}$ David A. Drew ${ }^{f}$ Mark J. Sarnak ${ }^{f}$ \\ Jeffrey M. Burns ${ }^{b, d}$ \\ ${ }^{a}$ Division of Nephrology and The Kidney Institute, ${ }^{b}$ Alzheimer's Disease Center, ${ }^{c}$ Department of Biostatistics, and \\ ${ }^{\mathrm{d}}$ Department of Neurology, University of Kansas Medical Center, Kansas City, KS, e Division of Psychology, University \\ of Kansas, Lawrence, KS, and fDivision of Nephrology, Tufts Medical Center, Boston, MA, USA
}

\section{Keywords}

Cognitive impairment - Kidney transplant .

Montreal Cognitive Assessment

\begin{abstract}
Background: Cognitive impairment is common in kidney transplant (KT) recipients and affects quality of life, graft survival, morbidity, and mortality. Failure to identify patients with cognitive impairment can withhold appropriate and timely intervention. This study determines whether measured cognition with standard screening tools offers any advantage over perceived cognition in screening transplant patients for cognitive impairment. Methods: Cognition was assessed in $157 \mathrm{KT}$ recipients using the Montreal Cognitive Assessment (MoCA; measured cognition). In addition, transplant physicians and nurse coordinators were asked to rate transplant recipients' level of cognition after routine clinical interactions (perceived cognition). Physicians and nurses were blind to MoCA scores. Perceived cognition scores were compared to MoCA scores. Results: Perceived cognition scores fairly correlated with MOCA scores $(\gamma=0.24, p=0.001$
\end{abstract}

\section{KARGER}

(c) 2017 S. Karger AG, Basel

E-Mail karger@karger.com

www.karger.com/nef for physicians and $\gamma=0.33, p<0.0001$ for nurses). Physician scores moderately correlated with nurses scores ( $k=0.44$, $p<0.0001$ ). Clinical perception had a low accuracy for identifying patients with cognitive impairment (sensitivity $66 \%$ for physicians, $65 \%$ for nurses), and those without cognitive impairment (specificity $67 \%$ for physicians, $76 \%$ for nurses). Conclusion: Clinical perception is inaccurate at detecting cognitive impairment in KT recipients. Objective tests should be considered to screen KT recipients for cognitive impairment.

(c) 2017 S. Karger AG, Basel

\section{Introduction}

Cognitive impairment is common in kidney transplant (KT) recipients [1] and it negatively impacts allograft survival and mortality $[2,3]$. Compared with its prevalence in age-matched controls, cognitive impairment is increased in ESRD and affects up to $87 \%$ of patients on dialysis [4]. Further, although typically considered a problem of the old, younger KT recipients also 
have cognitive impairment $[1,5]$. Fortunately, cognitive and psychomotor functions have been shown to improve after kidney transplantation [5-9].

Despite data demonstrating prevalent cognitive impairment in the kidney disease population and a potential risk of worse patient outcomes, routine screening for cognitive impairment in KT recipients is generally not performed. Several tools for screening for cognitive impairment are available [10]; however, their use in nephrology and transplant clinics is limited, leading to under-recognition of cognitive impairment [4]. Despite validated cognitive screening tools, transplant centers frequently depend on subjective clinical assessment of patient cognition. Failure to recognize cognitive impairment is likely to delay intervention $[11,12]$, and risk patient safety [13].

The care of a KT recipient is a complex and laborious combination of clinic appointments, laboratory tests, polypharmacy, and medication and dietary adherence. Medical nonadherence is fairly common in KT, and is a major cause for rejection and graft loss $[14,15]$. Cognitive impairment can impair the understanding of instructions and the ability to follow them, ultimately leading to medical nonadherence $[16,17]$. Recognition of cognitive impairment can help tailor the complexity of terms used while delivering education and instructions to patients, provide additional support from transplant coordinators and pharmacists to help with medical adherence, and help in timely identification, involvement, and counseling of caregivers and surrogate decision makers $[18,19]$. Identification of cognitive impairment can help with prognostication and management of patients [20]. Large studies have shown that patients with unrecognized cognitive impairment are less likely to be evaluated for reversible causes [21].

Studies in other populations have indicated that the identification and documentation of cognitive impairment by physicians, unaided by an objective screening tool remain poor [22]. It remains unclear if the subjective assessment of cognition by physicians or nurses is as reliable as an objective assessment in KT recipients. In this study, we evaluated the accuracy of transplant physicians and nurses' perception of KT recipients' cognition compared to a standard screening tool.

\section{Methods}

\section{Study Population}

Adult KT recipients presenting for outpatient post-transplant care at the University of Kansas Medical Center between June 29, 2015 and January 1, 2017 were screened for cognitive impairment by using the Montreal Cognitive Assessment (MoCA) [23] as part of their clinical care. After the clinic visit, one of the 6 physicians and 1 nurse practitioner (referred to as "physicians" hereafter), and one of the 9 nurse coordinators (referred to as "nurses" hereafter), who assessed the patient that day, scored the patients' cognition. The physicians and nurses were blinded to the MoCA results. Data collection was initiated as a quality improvement project to measure cognition in KT recipients and was declared Institutional Review Board exempt. Informed consent was therefore not obtained. Data were collected on paper case report forms and then entered into electronic case report forms using Research Electronic Data Capture, a web-based, electronic data capture tool hosted on a secure, password protected, HIPAA-compliant server [24]. To minimize the impact of high-dose steroids and operative procedures on cognition, all cognitive assessments were performed at least 1 month after KT. As part of a standardized protocol, all patients were maintained on a mycophenolic acid compound and a calcineurin inhibitor with or without prednisone.

Adult English-speaking KT recipients were included. Patients were excluded if they had (i) any hearing or visual impairment that would prohibit them from participating in MoCA; (ii) if they were unable to read, write, speak, or understand English; (iii) if they had stoke, concussion, or traumatic brain injury within 2 months of the evaluation; (v) if they already had a diagnosis of dementia; or (iv) if they had uncontrolled psychosis or active seizure disorder. Also, patients with psychiatric diseases or on psychotropic medications were not selected for MoCA.

\section{Standardized Screening for Cognitive Impairment}

Cognitive function was assessed with the MoCA (online suppl. data 1; for all online suppl. material, see www.karger.com/ doi/10.1159/000481182) [23]. The MoCA is a validated, clinicbased tool that samples from various domains of cognition and is sensitive in detecting mild cognitive impairment. MoCA consists of a single-page test with a maximum score of 30 (range $0-30$ ). The MoCA takes less than 10 min to complete and assesses 7 domains of cognition: visuospatial/executive, naming, memory (delayed recall), attention, language, abstraction, and orientation. The original English version 7.1 was used (http://www.mocatest.org/papertests/moca-test-full/). We chose MoCA over the more commonly used mini-mental state exam (MMSE) for detecting cognitive impairment, as the latter is more focused on memory rather than domains such as executive function, that are more commonly affected in kidney disease [25]. Compared to MMSE, MoCA has a better ability to detect vascular dementia with a higher area under the curve [26].

The MoCA was administered by medical assistants who underwent an hour of training that included a detailed review of online instructions on MoCA along with practice sessions on mock patients. MoCA was administered in a private room to minimize distraction and assure confidentiality.

\section{Perceived Cognition as Assessed by Transplant Physicians and}

Nurses

During a clinic visit, patients were evaluated by a physician and a nurse. All physicians and nurses independently rated patients' perceived cognition on a 10 -point Likert scale $(1=$ severe dementia, $10=$ no cognitive impairment) after the completion of clinic visit. These physicians and nurses were blinded to the 
MoCA results as well as to scores of other physicians and nurses. The interpretations of the scores from 1 to 10 (1-2 indicated dementia; $3-5$, moderate cognitive impairment; $6-8$, mild cognitive impairment, and 9-10, no cognitive impairment) and the definitions of dementia, moderate cognitive impairment, mid cognitive impairment, and no cognitive impairment were provided to the physicians and nurses (online suppl. data 2) on the scoring sheet where they were asked to assess patients by circling a number between 1 and 10 on the Likert scale. Since the aim for the project was to assess the ability of physicians and nurses to identify patients with cognitive impairment based on their current experience and knowledge, the physicians and nurses were not provided any additional education on the detection of cognitive impairment.

\section{Statistical Analysis}

Demographic and clinical variables that can potentially influence cognitive function such as age, gender, ethnicity, level of education, body mass index, blood pressure, history of smoking, diabetes, cause of ESRD, serum hemoglobin, estimated glomerular filtration rate, time on dialysis prior to transplant, and time after transplant were abstracted through review of the patient's electronic medical record. Descriptive statistics (mean and standard deviation for continuous measures, and frequencies and relative frequencies for categorical measures) were calculated for these variables.

The MoCA scores were compared with the physicians' and nurses' perceived cognition scores using gamma statistics because the measurement scales were different ( $0-30$ for MoCA and 1-10 for perceived cognition). Gamma statistics provide an assessment of not only association but also the direction of that association by assessing directional concordance in a structure-free manner by testing for monotonic relationships. The interpretation of gamma statistics is similar to that of a nonparametric correlation coefficient. They are both scaled from -1 (for negative associations) to 1 (for positive associations). The physician scores were compared with the nurses' scores using gamma and weighted kappa statistics. Kappa and weighted kappa measure agreement (both assessments providing the same rating on an observation) beyond what would be expected if the scoring were performed at random. While values can theoretically range from -1 to 1 , typically values greater than zero are observed, indicating some level of agreement beyond that expected by chance. Interpretation of the $\kappa$ and $\gamma$ values was performed according to Landis and Koch. Values $<0.20$ are considered poor, $0.21-0.40$ as fair, $0.41-0.60$ as moderate, $0.61-0.80$ as substantial, and $0.81-1.00$ as very good [27]. Correlation coefficients were calculated for each domain of cognition assessed by MoCA, namely, visuospatial/executive function, naming, memory (delayed recall), attention, language, abstraction, and orientation.

The performance characteristics of perceived cognition scores by physicians and nurses were also calculated. The MoCA scores were dichotomized into 2 categories: cognitive impairment (score $<26)$ and no cognitive impairment (score $\geq 26$ ) based on published data [23]. The perceived scores were dichotomized into 2 categories: cognitive impairment (score of $<8$ ) and no cognitive impairment (score of 9 or 10). The sensitivity, specificity, positive predictive value, and negative predictive value of physicians' and nurses' perception for determination of cognitive impairment were calculated. For all analyses, a $\mathrm{p}<0.05$ was considered statistically sig- nificant. Analyses were performed using SAS version 9.4 (SAS Institute Inc., Cary, NC, USA 2002-2012) and R version 3.3.1 (R Core Team 2016).

\section{Results}

All but 4 patients who were approached completed the MoCA assessment. Two of the four patients did not offer a specific reason for not completing the MoCA, one patient had forgotten his eye glasses on the day of the assessment, and one patient was getting late for another clinic appointment. Thus, a total of 157 patients completed MoCA. Of these, $57 \%$ were men, $74 \%$ were white, mean age was $55 \pm 14.8$ years, mean time post-transplant was $5.7 \pm 5.1$ years, $37 \%$ had a bachelor's degree or higher, and $28 \%$ had some college education. Thirty percent of the patients had an MoCA score of $<26$. Physicians and nurses perceived 43 and $36 \%$ of the patients to be cognitively impaired respectively. Table 1 lists baseline demographic and clinical data of patients based on their measured and perceived cognition. Ninety-five percent of the patients had their cognition assessed both by the physicians and nurses. Five patients had their cognition assessed only by a physician and three patients had it assessed only by a nurse. The mean MoCA score was $26.6 \pm 2.9$, and the mean perceived score by a physician was $9 \pm 1.4$ and by a nurse was $8.6 \pm 1.6$.

There was a fair correlation between the measured cognition scores and perceived cognition scores for physicians $(\gamma=0.24,95 \%$ CI $0.10-0.39, p=0.001)$ and nurses $(\gamma=0.33,95 \%$ CI $0.19-0.46, p<0.0001$; Table 2$)$. The variability in perceived cognitive impairment by physicians and nurses, when compared to MoCA scores, did not follow a specific direction (Fig. 1). We then compared the perceived score with individual domain scores in the MOCA and found domain-specific differences in correlation. Perceived cognition had the highest correlation with the naming domain for physicians $(\gamma=0.56,95 \% \mathrm{CI}$ $0.28-0.85 ; p=0.0001)$ and nurses $(\gamma=0.72,95 \%$ CI $0.55-$ $0.89, p<0.001$; Table 2). Conversely, the correlation of perceived cognition with attention, abstraction, or orientation domains was not as robust.

Next, we compared the accuracy of perceived scores by the physicians and the nurses for predicting cognitive impairment as defined by an MoCA score of $<26$. The agreement between the physicians and the nurses was moderate with $(\kappa=0.437,95 \%$ CI $0.347-0.527, p<0.0001$; Table 2). The sensitivity, specificity, positive predictive value, and negative predictive values for perceived cogni- 
Table 1. Patient characteristics $(n=157)$

\begin{tabular}{|c|c|c|c|c|c|c|}
\hline & $\begin{array}{l}\text { cognitive } \\
\text { impairment } \\
(n=48)\end{array}$ & $\begin{array}{l}\text { no cognitive } \\
\text { impairment } \\
(n=109)\end{array}$ & $\begin{array}{l}\text { cognitive } \\
\text { impairment } \\
(n=65)\end{array}$ & $\begin{array}{l}\text { no cognitive } \\
\text { impairment } \\
(n=84)\end{array}$ & $\begin{array}{l}\text { cognitive } \\
\text { impairment } \\
(n=55)\end{array}$ & $\begin{array}{l}\text { no cognitive } \\
\text { impairment } \\
(n=97)\end{array}$ \\
\hline Age, years, mean \pm SD & $58.5 \pm 14.7$ & $53.35 \pm 14.7$ & $60.1 \pm 13.9$ & $50.9 \pm 14.5$ & $60.1 \pm 13.0$ & $51.9 \pm 15.2$ \\
\hline \multicolumn{7}{|l|}{ Gender, $n(\%)$} \\
\hline Caucasian & $35(72.9)$ & $81(74.3)$ & $46(70.8)$ & $65(77.3)$ & $41(74.5)$ & $72(74.2)$ \\
\hline African American & $11(22.9)$ & $19(17.4)$ & $15(23.1)$ & $12(14.3)$ & $13(23.6)$ & $15(15.5)$ \\
\hline Other race & $2(4.2)$ & $9(8.3)$ & $4(6.2)$ & $7(8.3)$ & $1(0.0)$ & $10(10.3)$ \\
\hline \multicolumn{7}{|l|}{ Education level, $n(\%)$} \\
\hline$\geq$ Bachelor's degree & $15(31.3)$ & $43(39.4)$ & $19(29.2)$ & $36(42.8)$ & $18(32.7)$ & $38(39.2)$ \\
\hline Some college & $12(25)$ & $31(28.4)$ & $17(26.2)$ & $25(29.8)$ & $9(16.4)$ & $33(34)$ \\
\hline Systolic & $140.8 \pm 18.9$ & $138.7 \pm 15.6$ & $143.5 \pm 19.5$ & $136.1 \pm 13.9$ & $142.9 \pm 18.9$ & $137.5 \pm 15.2$ \\
\hline Diastolic & $75.3 \pm 14.3$ & $76.2 \pm 12.5$ & $73.9 \pm 14.4$ & $77.5 \pm 11.7$ & $74.3 \pm 14.3$ & $76.8 \pm 12.4$ \\
\hline Serum hemoglobin, g/dL, mean \pm SD & $12.8 \pm 1.7$ & $13.1 \pm 1.8$ & $12.8 \pm 1.9$ & $13.2 \pm 1.7$ & $12.8 \pm 1.8$ & $13.1 \pm 1.8$ \\
\hline $\mathrm{eGFR}, \mathrm{mL} / \mathrm{min} / 1.73 \mathrm{~m}^{2}$, mean $\pm \mathrm{SD}$ & $50.4 \pm 14.5$ & $50.32 \pm 13.3$ & $50.1 \pm 14.2$ & $50.6 \pm 13.3$ & $50.8 \pm 13.1$ & $50.0 \pm 14.2$ \\
\hline Time since KT, years, mean \pm SD & $7.0 \pm 4.9$ & $5.0 \pm 5.1$ & $6.0 \pm 5.0$ & $5.0 \pm 4.2$ & $5.9 \pm 4.7$ & $5.5 \pm 5.4$ \\
\hline Time on dialysis, years, mean \pm SD & $2.3 \pm 3.4$ & $2.8 \pm 3.3$ & $3.4 \pm 4.8$ & $2.1 \pm 1.1$ & $3.05 \pm 3.19$ & $2.32 \pm 3.48$ \\
\hline H/o smoking, $n(\%)$ & $26(54.2)$ & $42(38.5)$ & $32(49.2)$ & $32(38.1)$ & $29(52.7)$ & $36(37.1)$ \\
\hline H/o diabetes, $n(\%)$ & $25(52.1)$ & $29(26.6)$ & $27(41.5)$ & $24(28.6)$ & $23(41.8)$ & $30(30.9)$ \\
\hline
\end{tabular}

MoCA, montreal cognitive assessment; BMI, body mass index; KT, kidney transplantation; H/o, history of.

Table 2. Correlation of MoCA scores with the transplant physicians' and nurses' perceived scores

a Correlation of MoCA score (0-30) with the physician and nurses' perceived scores (1-10)

\begin{tabular}{|c|c|c|c|c|}
\hline & \multicolumn{2}{|c|}{ Correlation with physician scores } & \multicolumn{2}{|c|}{ Correlation with nurse scores } \\
\hline & correlation coefficient $(\gamma)$ & $95 \% \mathrm{CI}$ & correlation coefficient $(\gamma)$ & $95 \% \mathrm{CI}$ \\
\hline MoCA score $(0-30)$ & $0.24^{* *}$ & 0.10 to 0.39 & $0.35^{\mathrm{X}}$ & 0.19 to 0.46 \\
\hline
\end{tabular}

b Correlation of MoCA scores of different domains with the physician and nurses' perceived scores (1-10)

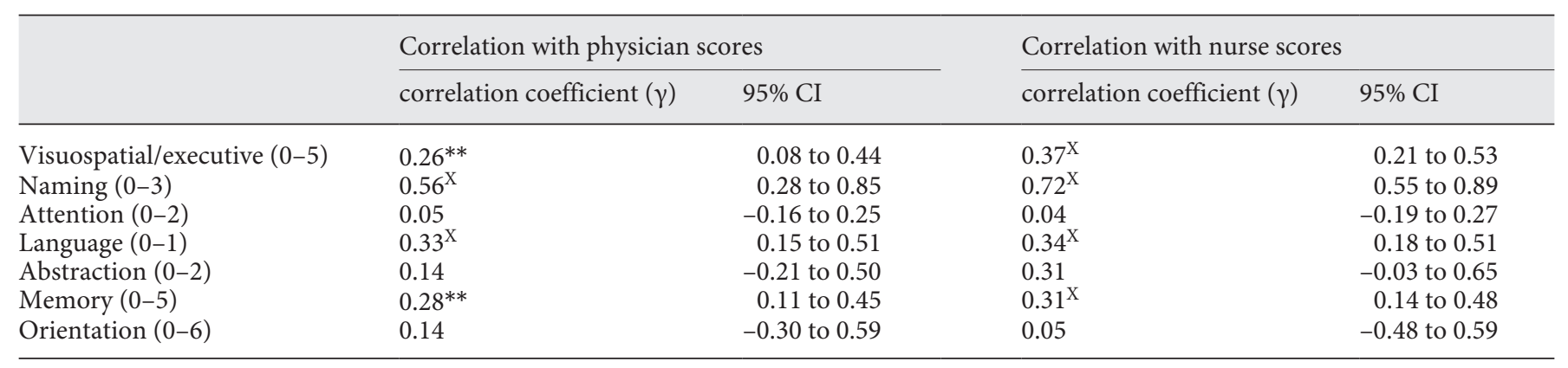

${ }^{* *} p$ value $<0.01 ; \mathrm{x} p$ value $<0.001$. 


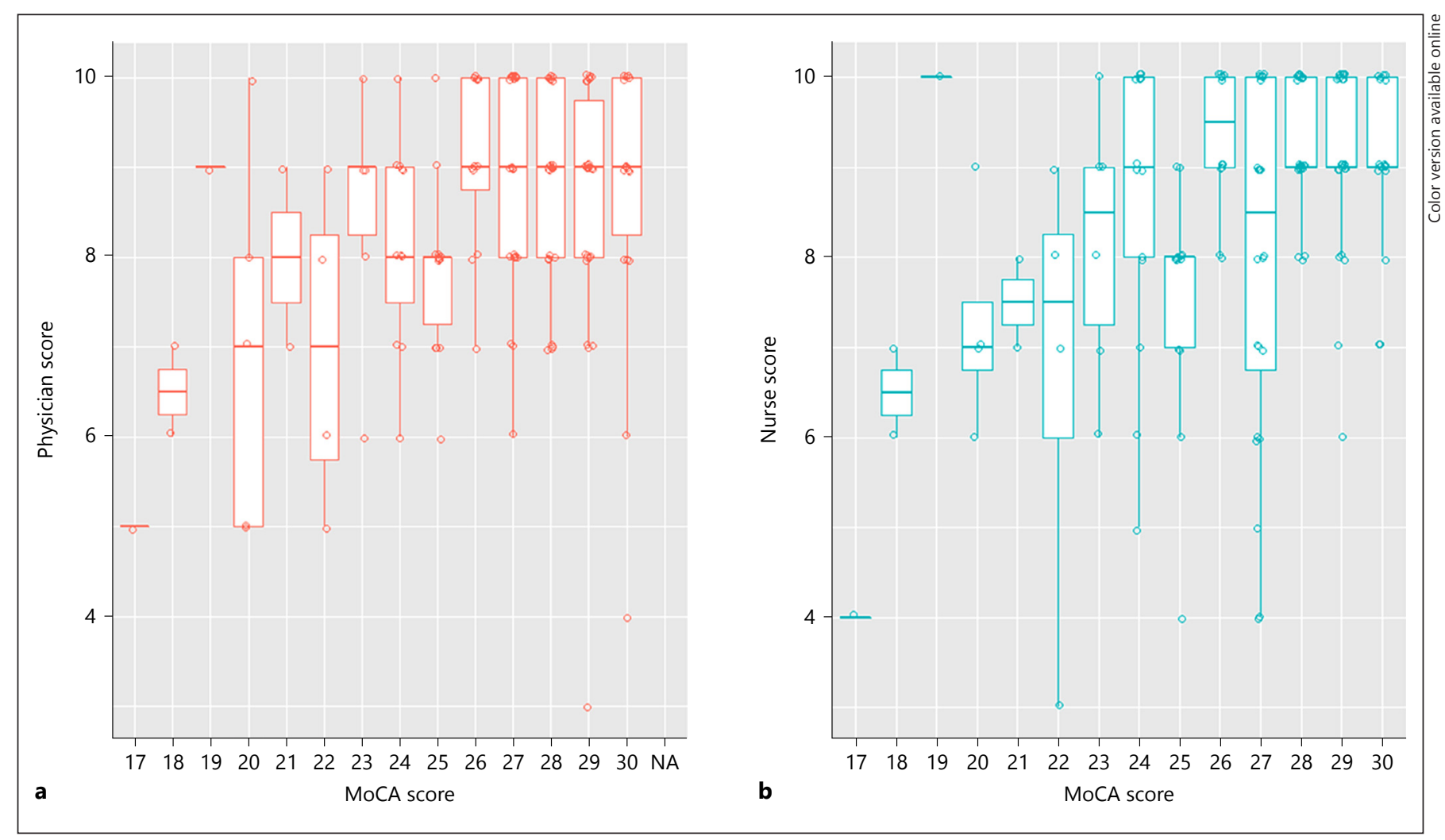

Fig. 1. Boxplot of MoCA scores and (a) physician and (b) nurse perceived scores. Perceived scores and MoCA scores are overlaid on a scatterplot of jittered data.

tion are shown in Table 3. The perceived cognition scores had a good negative predictive value for both physicians $(82 \%)$ and nurses (84\%), but poor sensitivity, specificity, and positive predictive value.

\section{Discussion}

We found that the correlation between perceived cognition of KT recipients by physicians and nurses and measured cognition was not good. In addition, there was a wide variability in the perceived cognition scores for each value of the MoCA score. Some domains of cognition, such as naming, correlated better than other domains with the perceived cognition. There was a moderate agreement between the cognitive assessment by the transplant physicians and nurses.

Our data suggest that physicians and nurses are not able to reliably identify KT recipients with cognitive impairment. This lack of reliability was bidirectional: many patients with normal MOCA scores were perceived to have cognitive impairment and other patients with abnormal
Table 3. Sensitivity, specificity, positive predictive value, and negative predictive value of physician perceived cognition score and nurse perceived cognition score in recognizing cognitive impairment

\begin{tabular}{lll}
\hline & $\begin{array}{l}\text { Physician } \\
\text { perceived } \\
\text { cognition score, \% }\end{array}$ & $\begin{array}{l}\text { Nurse } \\
\text { perceived } \\
\text { cognition score, \% }\end{array}$ \\
\hline Sensitivity & 65.9 & 65.2 \\
Specificity & 67.2 & 76.4 \\
Positive predictive value & 46.9 & 54.5 \\
Negative predictive value & 81.8 & 83.5 \\
\hline
\end{tabular}

MOCA scores were perceived to have normal cognition (Fig. 1). We also analyzed the performance characteristics by education, gender, and race (online suppl. data 3 ) and found that the sensitivity for perceived cognition by both physicians and nurses was lower for younger patients. It is possible that physicians and nurses were influenced in their ratings based on their historical interactions with the patients. Conversely, one would expect that repeated inter- 
actions helped the physicians and nurses to predict cognitive impairment more accurately. A plausible explanation for poor accuracy in identifying patients with cognitive impairment may be that the physicians and nurses are distracted by medical conditions of the patients and therefore focus less on cognition. Similar results, indicating inadequate identification of cognitive impairment in other highrisk patient populations $[21,22,28]$ indicate a need for objective assessments of cognition rather than relying on physician or nurse perception. Alternatively, physicians and nurses may lack education on detection of cognitive impairment, and education and awareness on cognitive impairment may improve the accuracy of perceived cognition, as it does in other populations [29].

We analyzed the physician and nurses scores separately, as they may have different kinds of interactions with patients. This also helps us identify variability in perceived cognition among different health care providers. Despite a poor correlation between measured and perceived cognition, there was a moderate agreement between physicians and nurses perceived cognition scores. This may indicate that patient factors rather than provider factors may play an important role in perception of cognition. For instance, post hoc analyses suggest that the sensitivity of health care providers in predicting cognitive impairment was much lower in younger than older patients -50 vs. $78 \%$ (online suppl. data 3 ). Although not powered, these observations suggest that both physicians and nurses perceive younger patients to be less likely to have cognitive impairment, simply because cognitive impairment is not suspected in younger patients. Further, the domain of naming correlated better with measured cognition for both physicians and nurses (Table 2). It is possible that naming as a domain is easier to evaluate during routine clinic visits and thereby correlate better with measured cognition. However, the domains of memory and executive function are likely better determinants of medical adherence but perhaps more difficult to assess during routine clinic visits, leading to a false estimation of global cognition by physicians and nurses.

To the best of our knowledge, this is the first study to assess whether perceived cognition by physicians and nurses correlates with measured cognition using a standard test in KT recipients. Our study is relevant because other studies have indicated a high prevalence of cognitive impairment in KT recipients [1] and association of poor cognition with inferior graft outcomes [2], perhaps through affecting medical adherence $[14,15]$. Nonadherence in patients may not be by patient's choice, but instead may be due to cognitive impairment. Cognitive im- pairment can further complicate the complex post-transplant management. Identification of patients with cognitive impairment is an important first step in the management of cognitive impairment. Failure to accurately identify patients with cognitive impairment can deprive them of additional support that could otherwise have been made available. This support may include repeated counseling, simplified verbal and written instructions, alarms or reminders, and perhaps more frequent monitoring. Cognitive impairment may also help identify patients with frailty, those who may benefit from additional support [30].

Our study does have some limitations. We did not perform a detailed neuropsychological assessment to follow screening by MoCA. We used MoCA because it is a clinic-based screening tool to assess cognition. Previous publications compared two screening tools, MMSE and MoCA, and found that the MoCA was more sensitive than MMSE for both mild cognitive impairment (sensitivity 90 vs. $18 \%$ ) and for mild Alzheimer's dementia (sensitivity 100 vs.78\%) with comparable specificity (87 vs. $100 \%$ ) [23]. MoCA is a practical test and can be used during routine clinic visits where detailed neuropsychological assessments are not readily available. We agree that further neuropsychological assessments should be performed in patients with abnormal MoCA scores. The Likert scale used for measuring perceived cognition needs psychometric validation. As a single-center study, with a majority of cohorts being white and with a high level of education, the generalizability of our results may be limited, but our data suggest that more studies are needed to investigate cognitive impairment in KT recipients.

In conclusion, our study found that physicians and nurses do not accurately screen patients with cognitive impairment. Because cognitive impairment affects medical adherence and post-transplant outcomes, objective tests are needed for an accurate assessment of cognition. Objective screening can appropriately identify patients with cognitive impairment so that appropriate interventions can be instituted.

\section{Acknowledgments}

We would like to thank nephrology physicians and nurse coordinators for their valuable contribution in providing perceived cognition scores. We would also like to thank our research coordinators, Baljit Kaur and Kenya Mitchell, and our medical assistants, Ariane Stanley, Gabie Barnard, Heather Miller, Kayla Turner, Meredith Michel, and Sarah Ishmael for performing the MoCA and for keeping the scores confidential. 


\section{Ethics Statement}

This study did not require informed consent nor review by the the University of Kansas Medical Center Institutional Review Board.

\section{Disclosure Statement}

None of the authors have any conflicts of interest to declare.

\section{Funding Source}

This study was supported in part by an NIH Clinical and Translational Science Award grant (UL1 TR000001, formerly UL1RR033179) awarded to the University of Kansas Medical Center, and internal support provided by KUMC Department of Medicine.

\section{References}

1 Gupta A, Mahnken JD, Johnson DK, Thomas TS, Subramaniam D, Polshak T, Gani I, John Chen G, Burns JM, Sarnak MJ: Prevalence and correlates of cognitive impairment in kidney transplant recipients. BMC Nephrol 2017; $18: 158$.

2 McAdams-DeMarco MA, Bae S, Chu N, Gross AL, Brown CHt, Oh E, Rosenberg P, Neufeld KJ, Varadhan R, Albert M, Walston J, Segev DL: Dementia and alzheimer's disease among older kidney transplant recipients. J Am Soc Nephrol 2017;28:1575-1583.

3 Sharma A, Yabes J, Al Mawed S, Wu C, Stilley C, Unruh M, Jhamb M: Impact of cognitive function change on mortality in renal transplant and end-stage renal disease patients. Am J Nephrol 2016;44:462-472.

4 Murray AM, Tupper DE, Knopman DS, Gilbertson DT, Pederson SL, Li S, Smith GE, Hochhalter AK, Collins AJ, Kane RL: Cognitive impairment in hemodialysis patients is common. Neurology 2006;67:216-223.

5 Gupta A, Lepping RJ, Yu AS, Perea RD, Honea RA, Johnson DK, Brooks WM, Burns JM: Cognitive function and white matter changes associated with renal transplantation. Am J Nephrol 2016;43:50-57.

6 Radić J, Ljutic D, Radić M, Kovačić V, DodigĆurković K, Šain M: Kidney transplantation improves cognitive and psychomotor functions in adult hemodialysis patients. Am J Nephrol 2011;34:399-406.

7 Griva K, Thompson D, Jayasena D, Davenport A, Harrison M, Newman SP: Cognitive functioning pre- to post-kidney transplantation-a prospective study. Nephrol Dial Transplant 2006;21:3275-3282.

8 Kramer L, Madl C, Stockenhuber F, Yeganehfar W, Eisenhuber E, Derfler K, Lenz K, Schneider B, Grimm G: Beneficial effect of renal transplantation on cognitive brain function. Kidney Int 1996;49:833-838.

9 Teschan PE, Ginn HE, Bourne JR, Ward JW: Neurobehavioral responses to "middle molecule" dialysis and transplantation. Trans Am Soc Artif Intern Organs 1976;22:190-194.

10 Pendlebury ST, Mariz J, Bull L, Mehta Z, Rothwell PM: MoCA, ACE-R, and MMSE versus the national institute of neurological disorders and stroke-Canadian stroke network vascular cognitive impairment harmonization standards neuropsychological bat- tery after TIA and stroke. Stroke 2012;43: 464-469.

11 Bradford A, Kunik ME, Schulz P, Williams SP, Singh H: Missed and delayed diagnosis of dementia in primary care: prevalence and contributing factors. Alzheimer Dis Assoc Disord 2009;23:306-314.

12 Larson EB, Reifler BV, Featherstone HJ, English DR: Dementia in elderly outpatients: a prospective study. Ann Intern Med 1984;100: 417-423.

13 Ball KK, Roenker DL, Wadley VG, Edwards JD, Roth DL, McGwin G Jr., Raleigh R, Joyce JJ, Cissell GM, Dube T: Can high-risk older drivers be identified through performancebased measures in a Department of Motor Vehicles setting? J Am Geriatr Soc 2006;54: 77-84.

14 Jindal RM, Joseph JT, Morris MC, Santella RN, Baines LS: Noncompliance after kidney transplantation: a systematic review. Transplant Proc 2003;35:2868-2872.

15 Garcia MF, Bravin AM, Garcia PD, Contti MM, Nga HS, Takase HM, de Andrade LG: Behavioral measures to reduce non-adherence in renal transplant recipients: a prospective randomized controlled trial. Int Urol Nephrol 2015;47:1899-1905.

16 Stilley CS, Bender CM, Dunbar-Jacob J, Sereika S, Ryan CM: The impact of cognitive function on medication management: three studies. Health Psychol 2010;29:50-55.

17 Insel K, Morrow D, Brewer B, Figueredo A: Executive function, working memory, and medication adherence among older adults. J Gerontol B Psychol Sci Soc Sci 2006;61:P102P107.

18 Arlt S, Lindner R, Rosler A, von RentelnKruse W: Adherence to medication in patients with dementia: predictors and strategies for improvement. Drugs Aging 2008;25: 1033-1047.

19 Ibrahim JE, Anderson LJ, MacPhail A, Lovell JJ, Davis MC, Winbolt M: Chronic disease self-management support for persons with dementia, in a clinical setting. J Multidiscip Healthc 2017;10:49-58.

20 Avila-Funes JA, Amieva H, Barberger-Gateau P, Le Goff M, Raoux N, Ritchie K, Carriere I, Tavernier B, Tzourio C, Gutierrez-Robledo LM, Dartigues JF: Cognitive impairment improves the predictive validity of the pheno- type of frailty for adverse health outcomes: the three-city study. J Am Geriatr Soc 2009;57: 453-461.

21 Callahan CM, Hendrie HC, Tierney WM: Documentation and evaluation of cognitive impairment in elderly primary care patients. Ann Intern Med 1995;122:422-429.

22 Chodosh J, Petitti DB, Elliott M, Hays RD, Crooks VC, Reuben DB, Galen Buckwalter J, Wenger N: Physician recognition of cognitive impairment: evaluating the need for improvement. J Am Geriatr Soc 2004;52:1051-1059.

23 Nasreddine ZS, Phillips NA, Bedirian V, Charbonneau S, Whitehead V, Collin I, Cummings JL, Chertkow H: The Montreal Cognitive Assessment, MoCA: a brief screening tool for mild cognitive impairment. J Am Geriatr Soc 2005;53:695-699.

24 Harris PA, Taylor R, Thielke R, Payne J, Gonzalez N, Conde JG: Research electronic data capture (REDCap) - a metadata-driven methodology and workflow process for providing translational research informatics support. J Biomed Inform 2009;42:377-381.

25 Sarnak MJ, Tighiouart H, Scott TM, Lou KV, Sorensen EP, Giang LM, Drew DA, Shaffi K, Strom JA, Singh AK, Weiner DE: Frequency of and risk factors for poor cognitive performance in hemodialysis patients. Neurology 2013;80:471-480.

26 Freitas S, Simoes MR, Alves L, Vicente M, Santana I: Montreal cognitive assessment (MoCA): validation study for vascular dementia. J Int Neuropsychol Soc 2012;18:1031-1040.

27 Landis JR, Koch GG: The measurement of observer agreement for categorical data. Biometrics 1977;33:159-174.

28 Lopponen M, Raiha I, Isoaho R, Vahlberg T, Kivela SL: Diagnosing cognitive impairment and dementia in primary health care - a more active approach is needed. Age Ageing 2003; 32:606-612.

29 Pond CD, Mant A, Kehoe L, Hewitt H, Brodaty $\mathrm{H}$ : General practitioner diagnosis of depression and dementia in the elderly: can academic detailing make a difference? Fam Pract 1994;11:141-147.

30 McAdams-DeMarco MA, Tan J, Salter ML, Gross A, Meoni LA, Jaar BG, Kao WH, Parekh RS, Segev DL, Sozio SM: Frailty and cognitive function in incident hemodialysis patients. Clin J Am Soc Nephrol 2015;10:2181-2189. 